\title{
Effect of topical application of two Polygala tenuifolia species decoction on guinea pig skin
}

\author{
Mingsan Miao*, Jingjing Shi, Lin Guo, Tan Wang, Weiyun Xin and Ming Bai \\ Department of Pharmacology, Henan University of Traditional Chinese Medicine, Zhengzhou, Henan, 450046, China
}

*For correspondence: Email: mingsanmiao_28@126.com Tel: +86-13203856889; Fax: +86-037165962546

Received: 4 September 2016

Revised accepted: 16 December 2016

\begin{abstract}
Purpose: To investigate the effect of the topical decoction of Polygala tenuifolia Willd. and Polygala sibirica $L$. on a guinea pig model of sore and ulcer.

Method: A guinea pig model of sore and ulcer was produced by Staphylococcus aureus, and 80 guinea pigs were evenly divided into eight groups, consisting of five males and five females in each group. Another 10 (5 male and 5 female) guinea pigs were used as the control group. The corresponding liquid medicine was applied to the ulcer site of the animals in each group, and administered the medicine for 6 $h$ daily with a continuous applicator for $12 \mathrm{~d}$. Lysozyme content in serum was measured to determine its effects on body tissue morphology.

Results: Compared with the control group, the sore skin score of guinea pigs in each dose group of decoction of Polygala tenuifolia Willd. and Polygala sibirica L. was significantly decreased. The skin showed apparent positive pathological changes and serum lysozyme concentration increased significantly $(p<0.05)$.

Conclusion: Decoctions of the two Polygala tenuifolia species exert a good therapeutic effect on the guinea pig model of sore and ulcer for topical use.
\end{abstract}

Keywords: Polygala species, Topical application, Guinea pig, Skin diseases, Lysozyme, Decoction

Tropical Journal of Pharmaceutical Research is indexed by Science Citation Index (SciSearch), Scopus, International Pharmaceutical Abstract, Chemical Abstracts, Embase, Index Copernicus, EBSCO, African Index Medicus, JournalSeek, Journal Citation Reports/Science Edition, Directory of Open Access Journals (DOAJ), African Journal Online, Bioline International, Open-J-Gate and Pharmacy Abstracts

\section{INTRODUCTION}

Pyogenic infections are caused by various types of pathogenic factors. Ancient physicians believe that skin and external diseases are mainly caused by Qi-stagnation and blood stasis, weakness of $Q i$ and blood, and toxic pathogens [1]. There are two sources of Polygala tenuifolia: one is the root of Polygala tenufiolia Willd. and the other is the root of Polygala sibirica L. Polygala tenuifolia has the functions of eliminating phlegm, calming the mind, and detumescence [2]. It can be used in the treatment of hypomnesis caused by heart-kidney disharmony, palpitation, sores, and other symptoms. Existing research shows that the chemical composition of Polygala tenuifolia contains saponin [3], which can have the functions as antitussives and expectorants, sedatives, and anti-aging tonics, and can cure sores and swellings [4]. It is recorded in a Chinese ancient medical work San Yin Fang that: "it is able to cure ulcer, boils, poisoning. Grinding Polygala tenuifolia into powders and mixing with a small cup of wine can be applied to the diseased area". However, studies of the modern clinical use of Polygala tenuifolia are rare. Lysozyme which acts exclusively on the B-1,4 glycosides of peptide [5], can cause cell death. It exerts antibacterial and anti-inflammatory effects [6]. Jingwanhong ointment, as a compound traditional Chinese medicine ointment for an 
external use, is widely used in the treatment of burn and ulcer. As Jingwanhong ointment mainly contains Sanguisorba officinalis, Gardenia, myrrh, Chinese rhubarb, pangolin, Angelica sinensis etc., and it has the function of detoxifying and draining pus [7], it is therefore widely used in surgery. This paper reported the effect of Polygala tenuifolia decoction in external use on a guinea pig model of sore and ulcer.

\section{EXPERIMENTAL}

\section{Drugs and reagents}

Drugs and reagents used were as follows: formaldehyde (Yantai Shuangshuang Chemical Co., Ltd, batch number: 20100308), Jingwanhong (Tianjin Darentang Jingwanhong Pharmaceutical Co., Ltd, batch number 211717), Staphylococcus aureus (supplied by the microbiology laboratory, Henan College of Traditional Chinese Medicine), sodium chloride injectable solution (Zhengzhou Yonghe Pharmaceutical Co., Ltd, batch no. 100422052), Qiaomier depilatory cream (Guangzhou Yibai Ya Cosmetics Co., Ltd, batch number 20100420), and lysozyme test kit (Nanjing Jiancheng Biological Engineering Research, batch no. 20101129).

\section{Plant materials}

Polygala tenuifolia Willd. was purchased from Jixian County, Tianjin. Polygala sibirica L. was obtained from sources in the mountains of western Jixian County. Polygala tenuifolia Willd. was identified to be the root of Polygala tenufiolia Willd. while Polygala sibirica L. is identified as the root of Polygala sibirica L.

Polygala decoction was prepared in the following procedure: $600 \mathrm{~g}$ Polygala tenuifolia Willd. was soaked in distilled water (10 times of the medicine) for $30 \mathrm{~min}$. Afterwards, the solution was simmered for $60 \mathrm{~min}$ and then filtered. After adding distilled water (eight times of the medicine) to simmer the solution for $60 \mathrm{~min}$ and filter the decoction, the filtrate obtained in the two times was merged and heated to $70{ }^{\circ} \mathrm{C}$ to further concentrate the crude drug content to $1.5,1$, and $0.5 \mathrm{~g} / \mathrm{mL}$. This was followed by high-temperature sterilisation and disinfection. The decoction of Polygala sibirica L. was prepared using the same method aforementioned.

\section{Instruments}

A TDL-40B centrifuge (Shanghai Anting Scientific Instrument Factory) and a MJ-180B mould incubator (Shanghai Yuejin Medical Instruments Factory) were used in the experiment.

S. aureus liquid was cultivated at $37^{\circ} \mathrm{C}$ in an agar medium for $24 \mathrm{~h}$. Then, sterile sodium chloride was injected in the $S$. aureus bacterial colony. The product was ground. In this way, a bacterial liquid containing 50 million $S$. aureus per ml was produced.

\section{Animals}

In the experiments, 90 guinea pigs (half male and half female) weighing $200 \sim 230 \mathrm{~g}$ were bought from Henan Kangda Laboratory Animal Co., Ltd (animal certificate no. 007267). Ethical approval was obtained from the Animal Experimental Center of Henan University of Traditional Chinese Medicine for animal studies (approval no. DWLL20130023). The experimental animals were treated in accordance with the 3R principles (reduction, replacement and refinement), proposed by International Laboratory Animal Science [8].

\section{Model establishment}

In the experiment, 90 guinea pigs (45 males and 45 female) weighing $200-250 \mathrm{~g}$ were used. The hair on an area of $3 \mathrm{~cm} \times 3 \mathrm{~cm}$ on the right rump of the guinea pigs was cut, along with the application of a light coating of Qiaomier depilatory cream. After $10 \mathrm{~min}$, the depilatory cream was washed off with warm water, and the guinea pigs did not show obvious damages on the skin with hair being removed. One day after hair removal, a group of 80 (half male and female) guinea pigs were randomly selected, with the other 10 guinea pigs forming the control group. The guinea pigs were anaesthetised by peritoneal injection of $10 \%$ chloral hydrate. Then, a cruciate and deep subcutaneous incision in a uniform size of $1 \mathrm{~cm} \times 1 \mathrm{~cm}$ was produced by using a razor blade in the part subjected to hair removal. If it bled, the wound was treated through press pressure hemostasis using sterile cotton. Thereafter, $S$. aureus bacteria (5 million $/ \mathrm{mL}, 0.5 \mathrm{~mL}$ ) was injected in each wound with a syringe once a day for three consecutive days until mild soreness appeared around the wound and purulent secretions were exuded from the wound.

\section{Animal grouping and administration}

One day after the hair removal, according to the principle of each group containing equal number of male and female guinea pigs, 80 guinea pigs which were established successful models, were randomly divided into eight groups: a model 
group (MG), a group administrated with Jingwanhong (Jingwanhong group, Jwh G), groups with high, medium, and low-doses of Polygala tenuifolia decoction (HD-Polygala tenuifolia Willd. DG, MD-Polygala tenuifolia Willd. DG, LD-Polygala tenuifolia Willd. DG), and groups with high, medium, and low-doses of Polygala sibirica L. decoction (HD-Polygala sibirica L. DG, MD-Polygala sibirica L. DG, LDPolygala sibirica L. DG). Each group contained 10 guinea pigs (half male and half female). The other 10 guinea pigs were included in the control group (CG, half male and female).

Corresponding solutions were applied to each group with two layers of gauze over the wound. To be specific, $0.5 \mathrm{~mL} 0.9 \%$ sodium chloride were injected in the MG and CG groups, while guinea pigs in Jwh $G$ group were subjected to Jingwanhong ointment $(0.3 \mathrm{~g}$ per animal, dressing volume $2 \mathrm{~cm} \times 2 \mathrm{~cm} \times 2 \mathrm{~mm}$ ). As for the groups with high, medium, and low-doses of Polygala tenuifolia decoction, they were administrated with $0.5 \mathrm{~mL}$ corresponding decoction $(0.75,0.5$, and $0.25 \mathrm{~g}$ per animal in high, medium, and low-dose groups). The groups with high, medium, and low-doses of Polygala sibirica L. decoction, were subjected to $0.5 \mathrm{~mL}$ of corresponding decoction $(0.75,0.5$, and $0.25 \mathrm{~g}$ per animal in high, medium, and low-dose groups respectively). The contact time (with the drug) was $6 \mathrm{~h}$ every day, with continuous coating for 12 d during administration. To avoid animals licking, biting, or nibbling the drugs on the wound, a separator was put in their cage.

\section{Determination of detection index}

From the first day of treatment, the animals were scored every third day to observe wound healing. At the $12^{\text {th }} \mathrm{d}$, the guinea pigs were anesthetised by $10 \%$ chloral hydrate via intraperitoneal injected $1 \mathrm{~h}$ after the administration. Afterwards, chests were opened to expose the hearts. Then, a $10 \mathrm{~mL}$ syringe was inserted in the hearts to extract $5 \sim 8 \mathrm{~mL}$ blood specimen via left ventricle. The blood specimens were centrifuged at $3,000 \mathrm{rpm}$ for $10 \mathrm{~min}$ to extract the serum according to the instructions in the LZM kit, to determine the content of lysozyme therein. The wounded skin was cut, fixed with $10 \%$ formaldehyde solution, subjected to HE staining, and observed under a light microscope to detect sore skin lesions.

The wound of ulcer skin was judged and scored according to the following standard:
0 Point: the ulcer skin was normal; 1 point: the ulcer skin was mild redness and swelling, and the wound shrank or was healed; 2 point: the skin was moderate redness and swelling, and there was a scab or a pus scab being found in the wound, or a small amount of purulent fluid being exuded from the wound; 3 point: the skin was serious redness and swelling, and there was a scab or a pus scab in the wound, or a small amount of purulent fluid being exuded from the wound.

\section{Statistical analysis}

The SPSS 14.0 statistical software for Windows was used for data analysis. The measurement results were expressed as "mean \pm standard deviation (SD)". Group comparison was carried out using the least using square difference (LSD) method. Riddit test showed that the Grade data were statistically significant. Differences were deemed statistically significant at $p<0.05$.

\section{RESULTS}

Effect of the decoction of Polygala tenuifolia Willd. and Polygala sibirica L. on ulcer skin score

Compared with the control group, at the $3^{\text {rd }}, 6^{\text {th }}$, $9^{\text {th }}$, and $12^{\text {th }}$ days, scores of the model group were increased significantly $(p<0.01)$, showing that using the method is feasible to prepare sore and ulcer models. Compared with the model group, until the $9^{\text {th }}$ day, the sore and ulcer skin scores in HD-Polygala tenuifolia Willd. DG, MDPolygala tenuifolia Willd. DG, and LD- Polygala sibirica L. DG were significantly reduced $(p<$ $0.05)$; at the $12^{\text {th }}$ day, ulcer score reduced significantly in the Jwh group, and in all groups with different doses of Polygala tenuifolia Willd. and Polygala sibirica L. decoction $(p<0.01, p<$ 0.05). The results are shown in Table 1.

\section{Effect of the decoction of Polygala tenuifolia Willd. and Polygala sibirica L. on serum lysozyme content}

Compared with the control group, serum lysozyme content of the guinea pigs in the model group significantly decreased $(p<0.01)$, indicating that the model was successfully replicated. Compared with the model group, serum lysozyme content increased significantly in the Jwh group, and all groups with different doses of Polygala tenuifolia Willd. decoction and Polygala sibirica L. decoction $(p<0.01$, see Table 2). 
Table 1: Effect of Polygala tenuifolia Willd. and Polygala sibirica L. decoction on skin score

\begin{tabular}{lcccc}
\hline Group & $\mathbf{3}^{\text {rd }}$ day & $\mathbf{6}^{\text {th }}$ day & $\mathbf{9}^{\text {th }}$ day & $\mathbf{1 2}^{\text {th }}$ day \\
\hline CG & $0.0 \pm 0.0$ & $0.0 \pm 0.0$ & $0.0 \pm 0.0^{* *}$ & $0.0 \pm 0.0^{\star *}$ \\
MG & $2.7 \pm 0.5^{\star *}$ & $2.6 \pm 0.5^{\star *}$ & $2.5 \pm 0.5$ & $2.1 \pm 0.3$ \\
Jwh G & $2.5 \pm 0.5$ & $2.4 \pm 0.5$ & $1.7 \pm 0.5^{\star *}$ & $1.3 \pm 0.5^{\star *}$ \\
HD-Polygala tenuifolia Willd. DG & $2.6 \pm 0.5$ & $2.5 \pm 0.5$ & $1.9 \pm 0.6^{*}$ & $1.5 \pm 0.5^{\star *}$ \\
MD-Polygala tenuifolia Willd. DG & $2.6 \pm 0.5$ & $2.5 \pm 0.5$ & $1.9 \pm 0.6^{*}$ & $1.5 \pm 0.5^{\star *}$ \\
LD-Polygala tenuifolia Willd. DG & $2.6 \pm 0.5$ & $2.5 \pm 0.5$ & $2.0 \pm 0.7$ & $1.5 \pm 0.5^{\star *}$ \\
HD- Polygala sibirica L. DG & $2.6 \pm 0.5$ & $2.5 \pm 0.5$ & $2.0 \pm 0.6$ & $1.5 \pm 0.5^{\star *}$ \\
MD- Polygala sibirica L. DG & $2.7 \pm 0.5$ & $2.6 \pm 0.5$ & $2.0 \pm 0.7$ & $1.5 \pm 0.5^{\star *}$ \\
LD- Polygala sibirica L. DG & $2.6 \pm 0.5$ & $2.5 \pm 0.5$ & $1.9 \pm 0.7^{*}$ & $1.6 \pm 0.5^{\star}$ \\
\hline
\end{tabular}

Note: Data are relative to those of physiological saline, compared with the model group, ${ }^{*} p<0.05$ and ${ }^{* *} p<0.01$ (mean $\pm \mathrm{SD}, n=10$, point)

Table 2: Effect on serum lysozyme content of Polygala tenuifolia and Polygala sibirica L. (mean \pm $\mathrm{SD}, \mathrm{n}=10$ )

\begin{tabular}{lc}
\hline Group & $\begin{array}{c}\text { Serum lysozyme } \\
\text { content }(\mu \mathrm{g} / \mathrm{ml})\end{array}$ \\
\hline CG & $38.03 \pm 5.28$ \\
MG & $16.26 \pm 4.78^{\star *}$ \\
Jwh G & $25.62 \pm 5.62^{* \star}$ \\
HD-Polygala tenuifolia & $28.60 \pm 6.36^{* *}$ \\
Willd. DG & \\
MD-Polygala tenuifolia Willd. & $26.75 \pm 4.28^{\star *}$ \\
DG & \\
LD-Polygala tenuifolia Willd. & $26.38 \pm 5.75^{\star *}$ \\
DG & $31.14 \pm 5.85^{\star *}$ \\
HD- Polygala sibirica L. DG & $27.18 \pm 6.4^{* *}$ \\
MD- Polygala sibirica L. DG & $27.54 \pm 6.98^{* *}$ \\
\hline LD- Polygala sibirica L. DG & $\begin{array}{l}\text { Note: Compared with physiological saline and the } \\
\text { control group, }{ }^{*} p<0.05 \text { and }{ }^{* *} p<0.01, \text { respectively }\end{array}$
\end{tabular}

Effect of Polygala tenuifolia Willd. and Polygala sibirica L. decoction on the pathological changes of guinea pig model of sore and ulcer

In the control group, subcutaneous tissue, skin tissue, and the dorsal squamous epithelium were normal (see Plate A in Figure 1). In the model group, much accumulation of inflammatory cells and abscesses was found in subcutaneous tissue, with thickening layers and dorsal squamous epithelium (see Plate B in Figure 1). In the Jwh group, subcutaneous tissue exhibited diffuse infiltration of inflammatory cells and multiple small abscesses, accompanying with thickening of the back of the keratinised layer and squamous epithelial hyperplasia (see Plate $C$ in Figure 1). In high doses of the Polygala tenuifolia Willd. group, subcutaneous tissue was found to show diffuse infiltration of inflammatory cells and granulation of tissue, a keratinised layer, and normal squamous epithelium of the rear (see Plate $D$ in Figure 1). With medium doses of Polygala tenuifolia Willd, subcutaneous tissue showed much inflammatory cell infiltration and granulation of tissue, thickening of the keratinised layer, and squamous epithelial hyperplasia on the rear (see Plate E in Figure 1). With low doses of Polygala tenuifolia Willd., subcutaneous tissue showed much inflammatory cell infiltration and multiple small abscesses formed with thickening of the keratinised layer, and squamous epithelial hyperplasia on the rear (see Plate $F$ in Figure 1). With high doses of Polygala sibirica L., inflammatory cell infiltration was found to be scattered in the subcutaneous tissue, and show granulation of tissue, thickening of the keratinised layer, and squamous epithelial hyperplasia on the rear (see Plate G, Figure 1). With medium doses of Polygala sibirica L., subcutaneous tissue showed multiple small abscesses and granulation of tissue, the keratinised layer was normal, as the dorsal squamous epithelial hyperplasia (see Plate $\mathrm{H}$, Figure 1). With low doses of Polygala sibirica L., subcutaneous tissue presented diffuse inflammatory cell infiltration and granulation of tissue, thickening keratinised layer, and squamous epithelial hyperplasia on the rear (see Plate I, Figure 1).

Through pathological observation, compared with the control group, the model group showed more serious pathological injury $(p<0.01)$, indicating that the sore and ulcer model was successfully established. Compared with the model group, groups with every dose of Polygala tenuifolia Willd. and Polygala sibirica L., and the Jwh group showed significant reduction in the ulceration while the guinea pigs underwent pathological changes $(p<0.01)$. The results indicated that the decoction of Polygala tenuifolia Willd. and Polygala sibirica L. contribute to heal ulcers in guinea pigs (see Table 3).

In Table 3, "-" indicates that the back, the skin of the epidermis, and the subcutaneous tissue 
presented normal squamous epithelium. "+" refers to that the back and the skin of the epidermis had normal squamous epithelium and a small amount of inflammatory cell infiltration was seen in the subcutaneous tissue. "++" means that the squamous epithelium of the back and the hyperplasia of the skin of the epidermis were normal and subcutaneous tissue showed a significant inflammatory cell infiltration and little scattering thereof. "+++" shows that the squamous epithelium of the back and the skin layer was significantly thickened, and a significant amount of inflammatory cell infiltration was seen with the formation of larger abscesses in the subcutaneous tissue.

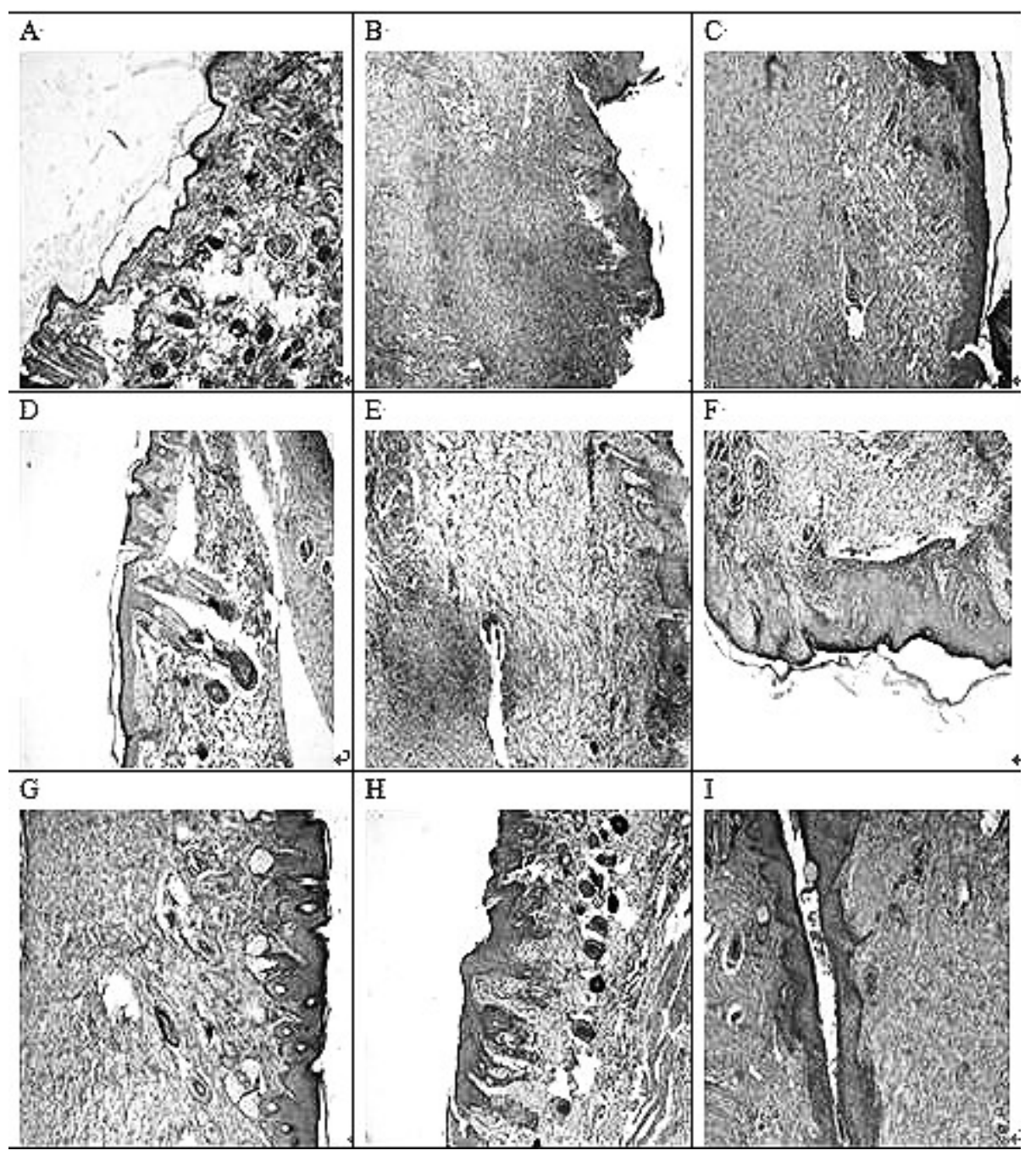

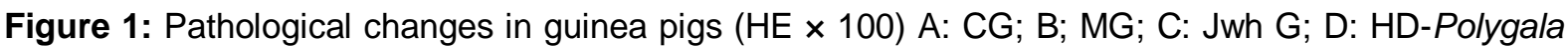
tenuifolia Willd. DG; E: MD-Polygala tenuifolia Willd. DG; F: LD-Polygala tenuifolia Willd. DG; G: HDPolygala sibirica L. DG; H: MD-Polygala sibirica L. DG; I: LD-Polygala sibirica L. DG

Table 3: Effect on the pathological changes of guinea pigs model of sore and ulcer (mean $\pm S D, n=10$ )

\begin{tabular}{lccccc}
\hline Group & - & + & +++ & $\mathbf{+ + +}$ & $\boldsymbol{P}$-value \\
\hline CG & 10 & 0 & 0 & 0 & $*$ \\
MG & 0 & 0 & 1 & 9 & $* *$ \\
Jwh G & 0 & 3 & 5 & 2 & $* *$ \\
HD-Polygala tenuifolia Willd. DG & 4 & 3 & 3 & 0 & $* *$ \\
MD-Polygala tenuifolia Willd. DG & 2 & 4 & 4 & 0 & $* *$ \\
LD-Polygala tenuifolia Willd. DG & 0 & 2 & 7 & 1 & $* *$ \\
HD- Polygala sibirica L. DG & 9 & 1 & 0 & 0 & $* *$ \\
MD- Polygala sibirica L. DG & 4 & 2 & 4 & 0 & $* *$ \\
LD- Polygala sibirica L. DG & 4 & 4 & 2 & 0 & \\
\hline
\end{tabular}

Note: Compared with physiological saline and the model group, ${ }^{*} p<0.05,{ }^{* *} p<0.01$ separately 


\section{DISCUSSION}

Sore and ulcer are mainly caused by Qi stagnation, stagnation of Ying-qi, and meridian obstruction [9]. Wound infection, ulcer formation, pain in the skin, and shallow wound infections are called abscesses. Sore and ulcer are skin diseases whose wound is found within the muscles, and the purulent blood stasis is serious after being infected, accompanied by swelling, heat, and pain in the skin [10]. Lysozyme, as one of the non-specific immune factors in human body [11], can relax bacterial cell wall which therefore cannot protect the cell, causing final cell death [12].

By observing the content of lysozyme in serum, it can more favourably reflect the antibacterial and anti-phlogistic effects of the drug. Therefore, content of lysozyme can be used as a depth index for determining the healing degree of ulcer. After skin ulcer occurs, marking scores for the wound can help to judge the development degree of ulcer and the improvement of the pathological changes can better reflect the sore from the microscopic aspect.

By generating skin injury artificially in guinea pigs through Staphylococcus aureus infection, this research established the guinea pig model of sore and ulcer. Results revealed that the modelling method was reasonable. Wound tenderness, purulent secretions, redness, pain, heat, and swelling were seen 2 days after the infection [13].

The experimental results showed that every dose used in Polygala tenuifolia Willd. and Polygala sibirica L. groups significantly reduced the skin ulcer score of guinea pigs and significantly increased serum lysozyme content of the pyocutaneous model therein. At the same time, the drugs significantly reduced pathological changes in the guinea pigs. The results showed that the two Polygala tenuifolia species have a treatment effect on sore and ulcer of guinea pigs, suggesting that they have a healing function.

\section{CONCLUSION}

The findings of this study show that Polygala tenuifolia Willd. and Polygala sibirica L. can promote wound healing improve serum lysozyme levels. These two species are sources of Polygala tenuifolia included in the 2010 Chinese Pharmacopoeia [14], and demonstrate good therapeutic effects on the guinea pig model of sore and ulcer. Further studies are, however, required to develop these formulations for clinical applications.

\section{DECLARATIONS}

\section{Acknowledgement}

This study was supported by National " $11^{\text {th }}$ FiveYear" Support Program (no. 2008BAI53B09); University Science and Technology Innovation Team (no. 2012IRTSTHN011); and Zhengzhou Science and Technology Innovation Team Project (no. 131PCXTD612). Besides, the authors would like to thank Ruiqin Li, Professor of Pathology, Henan University of Traditional Chinese Medicine for assisting with the pathology studies.

\section{Conflict of Interest}

No conflict of interest associated with this work.

\section{Contribution of Authors}

The authors declare that this work was done by the authors named in this article and all liabilities pertaining to claims relating to the content of this article will be borne by them.

\section{Open Access}

This is an Open Access article that uses a funding model which does not charge readers or their institutions for access and distributed under the terms of the Creative Commons Attribution License (http://creativecommons.org/licenses/by 14.0) and the Budapest Open Access Initiative (http://www.budapestopenaccessinitiative.org/rea d), which permit unrestricted use, distribution, and reproduction in any medium, provided the original work is properly credited.

\section{REFERENCES}

1. Yue $J H$, Zhang $Q H$, Sun ZR. The status quo and analysis of clinical studies of Chinese medicine for the external treatment of sores. J External Therapy Tradit Chinese Med 2013; 22(4): 55-56.

2. Dong $X B$, Li J, Zheng $Y$. Preparation of tenuifolin from radix polygalae as a reference substance. Tradit Chinese Drug Res Clin Pharmaco 2007; 18(1): 53-54.

3. Lin JK, Yan XP, Guan SJ, Li L. A study of the saponin content of different processed products of radix polygalae. Chinese J Exp Tradit Med Form 2011; 17(11): 89-91.

4. Du FL, Wang J. Research progress of Polygala. Huazhang 2012; (28): 319-320.

5. Dong $A X$, Wang $Q$, Fan XR, Wang $P$, Cui L, Yuan JG, Zhang JF. Immobilisation of lysozyme catalyzed by laccase on bast fabrics and its antibacterial properties. $J$ Funct Mater 2014; 2(45): 02044-02048. 
6. He JH, Liu Y, Liu GJ, Li LN, Li HC, Wang LJ. Application of, and research progress in, lysozyme in medicine. Prog Pharm Sci 2008; 18(2): 16-19.

7. Zhang SC, Guo DL, Liu WW, et al. Clinical application development of Jingwanhong Ointment. J Pharm Res, 2014, 33(12): 718-720.

8. Qv YQ, Ha HX, Guo YQ, et al. Application of $3 R$ principle in medical animal experimental work. Laboratory Animal Science, 2010, 27(6): 84-85.

9. Zhang GY, Li XJ. Overview of the study on the treatment of sores with external Chinese medicine. Glob Tradit Chinese Med 2010; 3(2): 101-106.

10. Tang PP, Guo XF, Bai Ming, Miao MS. Effect of Ampelopsis external application on a sores/ulcers model. China J Tradit Chinese Med Pharm 2012; 27(3): 702-705.

11. Tang SW. Research progress in the comprehensive application of egg shell and egg white. Shanghai J Anim Husband Vet Med 2014; 1: 20-22.

12. Zhang $P$, Jiang MF, Wang $Y$. Research progress of lysozyme from animal source. China J Biol Engrng, 2012, 32 (08): 87-93.

13. Yu SY, Miao MS. Effects of external application of physalis on sores and ulcers in a rats and guinea pigs. China J Tradit Chinese Med Pharm 2014; 29(2): 611614.

14. Liu YL, Wang YY, Zeng K. Hole identification of Polygala tenuifolia Willd. and Polygala. J Chinese Med, 2014, 29 (10): 1473-1476. 